\title{
Reducing the Momentum Spread of 8-GeV proton Beam via the Bunch Rotation in Booster
}

Xi Yang, Charles M. Ankenbrandt, Rene Padilla, William A. Pellico, Joseph E. Dey, and Kiyomi Koba

Fermi National Accelerator Laboratory

Box 500, Batavia IL 60510

\begin{abstract}
It requires Booster to be able to deliver $8-\mathrm{GeV}$ proton beams to Main Injector at the intensity of $4.5 \times 10^{12}$ per batch with a longitudinal emittance of $0.12 \mathrm{eV} \cdot \mathrm{sec}$ and a momentum spread $(\Delta \mathrm{p})$ of $18 \mathrm{MeV}$ in order to achieve the antiproton production rate of $24 \times 10^{10}$ per hour. Bunch rotation via the RFSUM reduction at the end of a cycle has been implemented to reach the goal. Afterward, it is important for us to develop diagnostic tools and tuning capabilities to make bunch rotation operationally reliable.
\end{abstract}

\section{Introduction}

In order to achieve the antiproton production rate of $24 \times 10^{10}$ per hour, it requires Booster to be able to deliver $8-\mathrm{GeV}$ proton beams to Main Injector at the intensity of $4.5 \times 10^{12}$ per batch with a longitudinal emittance of $0.12 \mathrm{eV} \cdot \mathrm{sec}$ and a momentum spread $(\Delta \mathrm{p})$ of 18 $\mathrm{MeV}$. The momentum spread of a nominal $8-\mathrm{GeV}$ proton beam at such beam intensity is about $30 \mathrm{MeV}$. Bunch rotation (BR) via the RFSUM reduction at the end of a cycle has been implemented to reduce the bucket height, so the $18 \mathrm{-MeV}$ momentum spread can be achieved before the beam is transferred from Booster to Main Injector.[1,2] RFSUM is the vector sum of rf voltages at cavity gaps of all Booster rf stations.

Usually, the momentum spread of $8-\mathrm{GeV}$ proton beam increases with the increase of the beam intensity. However, the limit of $\Delta \mathrm{p}=18 \mathrm{-MeV}$ is set by the momentum acceptance of slip stacking two Booster batches into one batch in Main Injector.[1] Any further reduction in $\Delta \mathrm{p}$ can potentially increase the antiproton stacking rate. It is 
important for us to optimize the BR process via the ESME simulation,[3] and also to develop diagnostic tools for making BR operationally reliable.

\section{Numerical Investigations}

ESME simulations are used to investigate the following questions: $1^{\text {st }}$, an optimal BR voltage $\left(V_{B R}\right)$, which provides the maximum reduction in $\Delta \mathrm{p}$ and at the same time doesn't cause any extra beam loss and nonlinear momentum tail in the longitudinal phase space (LPS), exists or not; $2^{\text {nd }}$, and the RFSUM curve before BR influences the minimum $\Delta \mathrm{p}$, which can be achieved after BR, or not.

In order to answer the $1^{\text {st }}$ question, three different BR voltages, $0.1 \mathrm{MV}, 0.2 \mathrm{MV}$, and $0.3 \mathrm{MV}$, are used in the ESME simulation while the rest of the RFSUM curve in a cycle is kept the same, as shown in Figs. 1(a) and 1(b). All the simulations were done at the beam intensity of $4.1 \times 10^{12}$, including the space charge effect. In the situation of $V_{B R}=0.1 \mathrm{MeV}$, the phase projection of the particle distribution in the LPS before BR is shown in Fig. 1(c), and the momentum projection is shown in Fig. 1(d). $\Delta$ p is nearly 40 $\mathrm{MeV}$. The phase and momentum projections at the optimal BR time are shown in Figs. 1(e) and 1(f) respectively. $\Delta \mathrm{p}$ after BR is less than $20 \mathrm{MeV}$. However, there are some particles outside the bucket area, which is indicated by the red contour in Fig. 1(g), and eventually they will get lost inside Booster. In the situation of $V_{B R}=0.2 \mathrm{MeV}$, the momentum projection and the particle distribution in the LPS at the optimal BR time are shown in Figs. 1(h) and 1(i) respectively. $\Delta \mathrm{p}$ is less than $20 \mathrm{MeV}$, and there is no beam loss. In the situation of $V_{B R}=0.3 \mathrm{MeV}$, the momentum projection at the optimal BR time is shown in Fig. 1(j). $\Delta \mathrm{p}$ is greater than $25 \mathrm{MeV}$.

It is clear that when $V_{B R}$ is too large, the reduction in $\Delta \mathrm{p}$ isn't sufficient for satisfying the requirement of momentum acceptance $\Delta \mathrm{p}=18-\mathrm{MeV}$; when $V_{B R}$ is too small, some of particles get lost during BR, and nonlinear momentum tails are likely to be developed during BR since the bunch gets comparably long in the rf bucket; there exists an optimal $V_{B R}$, which allows a sufficient reduction in $\Delta \mathrm{p}$ in order to satisfy the momentum acceptance, and at the same time, the phase projection occupies about onethird of the rf period, as shown in Fig.1(i), and there isn't any nonlinear momentum tail. 
In order to answer the $2^{\text {nd }}$ question, a different RFSUM curve, RFSUM 2, is used in the ESME simulation, as shown in Fig. 2(a). The phase and momentum projections of the particle distribution in the LPS right before BR are shown in Figs. 2(b) and 2(c). Compared to Figs. 1(c) and 1(d), the bunch gets longer in Fig. 2(b), and $\Delta \mathrm{p}$ is slightly more than $30 \mathrm{MeV}$ in Fig. 2(c) instead of nearly $40 \mathrm{MeV}$ in Fig. 1(d). These differences happen because smaller RFSUM voltages are used in RFSUM 2 during the time of $22 \mathrm{~ms}$ to $33.1 \mathrm{~ms}$ (right before BR) in a cycle. Usually, when the RFSUM voltages used in the acceleration are higher, the momentum spread of the beam will be larger and the bunch length will become shorter. $V_{B R}$ is optimized for RFSUM 2, and the result is $0.13 \mathrm{MeV}$ instead of $0.2 \mathrm{MeV}$ for the situation of Fig. 1(a). However, $\Delta \mathrm{p}$ after BR is the same with that in Fig. 1(h), about $18 \mathrm{MeV}$, as shown in Fig. 2(e).

Whenever some changes are made to the RFSUM curve, initial conditions, such as the momentum spread, and the bunch length, etc., for BR have been modified, and $V_{B R}$ should be optimized for the new situation in order to achieve $\Delta \mathrm{p}=18 \mathrm{-MeV}$.

\section{Diagnostic Tools}

The goal of $\mathrm{BR}$ is to reduce the momentum spread of $8-\mathrm{GeV}$ proton beam to 18 $\mathrm{MeV}$ without causing any longitudinal emittance (LE) growth. Since in the situation that there exists a mismatch between the beam and rf bucket centers, the beam will tumble inside the bucket and eventually occupy a bigger space with a grown LE, it is important for us to develop some diagnostic tools, which are able to observe the mismatch situation.

Since a synchronous phase detector (SPD) has been implemented in the Fermilab Booster for directly measuring the relative phase between the beam and RFSUM,[4] any change between the beam and $\mathrm{rf}$ bucket centers during BR (about a quarter of the synchronous period) will appear as the phase slew in the SPD signal. The data, as shown in Fig. 3(a), were taken when there was a BR-induced LE growth. The LE of 8-GeV beam is measured after the Booster-to-Main Injector transfer for both situations with BR on and off, and any difference between these two measurements indicates the BRinduced LE growth. The SPD and RFSUM signals are shown as the black and blue curves in Fig. 3(a). The red dash line indicates the trigger time for BR, and the maximum phase slew between the beam center and the RFSUM waveform during BR is greater than 30 degrees. 
The data, as shown in Fig. 3(b), was taken when there was a negligible BRinduced LE growth. The SPD and RFSUM signals are shown as the black and blue curves respectively. The maximum phase slew between the beam center and the RFSUM waveform during BR is about 10 degrees instead of 30 degrees in Fig. 3(a), and also RFSUM decreased much less than that in Fig. 3(a). Besides, the mountain range plot (MRP) can be used to diagnose the motion between the beam and the rf bucket centers. Since the MRP is designed to look the same beam bunch every $m$ Booster turns, any motion between the beam bunch and the RFSUM waveform will appear as the variation of the bunch position from trace to trace. Here, $m$ is the turn number to be escaped from trace to trace. When the phase slew between the beam and the rf waveform is comparably small, the bunch will stay at the similar position from trace to trace of the MRP. At the same time when the data in Fig. 3(b) were taken, the MRP was recorded during BR with the setup of $m=10$, as shown in Fig. 3(c). During BR, each bunch stayed nearly the same place in the MRP, except that the bunch became longer.

\section{BR Optimization}

It is unavoidable that the phase slew between the beam and the RFSUM waveform during BR will cause the LE growth of the beam. And this usually happens when RFSUM couldn't stay the constant value of $V_{B R}$ and keeps on decreasing, as shown in Fig. 3(a). The phase slew during BR is likely caused by the beam loading (BL) of rf cavities, since $\mathrm{BR}$ is performed via the RFSUM reduction through para-phasing the A and B stations.

The $\mathrm{BL}$ to $\mathrm{A}$ stations is identical to the $\mathrm{BL}$ to $\mathrm{B}$ stations during $\mathrm{BR}$, and as a result, the RFSUM vector is no longer aligned relative to the beam according to the desired synchronous phase. A mismatch between the beam center and the $\mathrm{rf}$ waveform is developed. The feedback system of rf stations tries to compensate the BL via correcting the phase error, except the correction is slow, in the order of tens of micro-seconds.

Since the process of para-phasing during BR can be controlled via a ramped paraphase curve,[5] the time, which is taken for RFSUM to be reduced from the nominal value to $V_{B R}$, can be flexibly adjusted to allow the feedback system to have sufficient time to compensate the $\mathrm{BL}$.

At the same time, instead of using the A and B balancers to make RFSUM from A stations $\left(\mathrm{V}_{\mathrm{A}}\right)$ equal to RFSUM from $\mathrm{B}$ stations $\left(\mathrm{V}_{\mathrm{B}}\right)$, the $\mathrm{BL}$ during $\mathrm{BR}$ can be 
compensated by individually adjusting $\mathrm{V}_{\mathrm{A}}$ and $\mathrm{V}_{\mathrm{B}}$. How this can be done is demonstrated in Figs. 4(a)-(d). $V_{A}$ and $V_{B}$ before BR are shown as the black and blue vectors in Fig. 4(a). Without considering the $\mathrm{BL}, \mathrm{V}_{\mathrm{A}}$ and $\mathrm{V}_{\mathrm{B}}$ during $\mathrm{BR}$ are shown as the dashed black and blue vectors in Fig. 4(b), and RFSUM $\left(\mathrm{V}_{\mathrm{RF}}\right)$, which is determined by $V_{B R}$, is shown as the orange vector. Taking the $\mathrm{BL}$ into consideration, the $\mathrm{BL}$ of $\mathrm{A}$ stations $\left(\mathrm{V}_{\mathrm{AL}}\right)$ and the $\mathrm{BL}$ of $\mathrm{B}$ stations $\left(\mathrm{V}_{\mathrm{BL}}\right)$ are represented by red dashed vectors in Fig. 4(c), RFSUM during BR becomes $V_{\mathrm{RFL}}$, which is represented by the green vector. Since $V_{\mathrm{RFL}}$ points to a direction different from $\mathrm{V}_{\mathrm{RF}}$, the quadruple oscillation (or called "tumble" before) is excited. Without making any change to the para-phasing curve during $\mathrm{BR}$, the amplitudes of $\mathrm{V}_{\mathrm{A}}$ and $\mathrm{V}_{\mathrm{B}}$ are adjusted via the $\mathrm{A}$ and $\mathrm{B}$ balancers to the new values of $\mathrm{V}_{\mathrm{a}}$ and $\mathrm{V}_{\mathrm{b}}$, as shown by the dotted black and blue vectors in Fig. 4(d), and the vector sum of $\mathrm{V}_{\mathrm{a}}, \mathrm{V}_{\mathrm{b}}, \mathrm{V}_{\mathrm{AL}}$, and $\mathrm{V}_{\mathrm{BL}}$ is equal to the desired bunch rotation voltage $V_{B R}$.

\section{Conclusions}

$\mathrm{BR}$ is desired to be done at the relatively stationary rf bucket. Any phase slew between the beam and rf waveform can be captured either by the SPD or by the MRP. The para-phase curve can be used for the detail tuning in order to keep $V_{B R}$ constant during $\mathrm{BR}$. Besides, the $\mathrm{A}$ and $\mathrm{B}$ balancers can be applied for the beam loading compensation during BR. There are a sufficient amount of diagnostic tools and tuning capabilities for optimizing BR in order to achieve the goal of $\Delta \mathrm{p}=18-\mathrm{MeV}$ and 0.12 $\mathrm{eV} \cdot \mathrm{sec}$ at the intensity of $4.5 \times 10^{12}$ per batch.

\section{References:}

[1] K. Koba, etc., "SLIP STACKING EXPERIMENTS AT FERMILAB MAIN INJECTOR”, FERMILAB-CONF-03-107.

[2] K. Koba and J. Steimel, “SLIP STACKING”, FERMILAB-CONF-02-205.

[3] http://www-ap.fnal.gov/ESME/.

[4] X. Yang, and R. D. Padilla, "A Synchronous Phase Detector for the Fermilab Booster", FERMILAB-TM-2234.

[5] http://garlic.fnal.gov/ cdrennan/LLRF-WWW/LLRF_Projects.htm. 


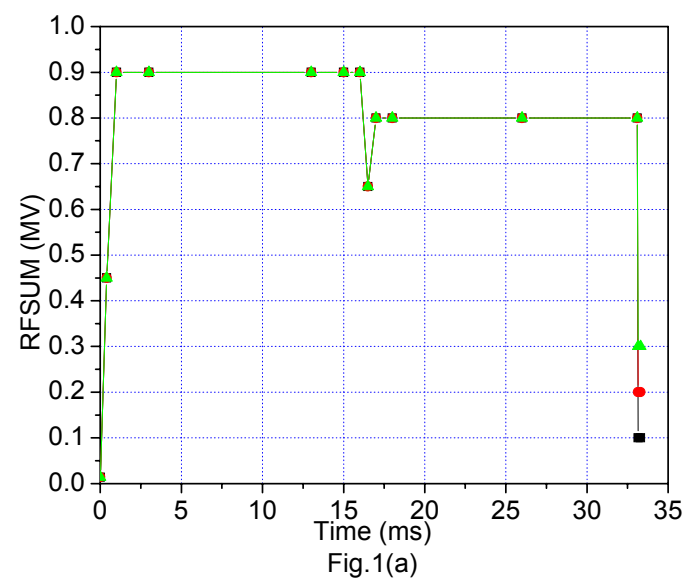

Itar 19600
$3.312 \mathrm{E}-02$ SEC

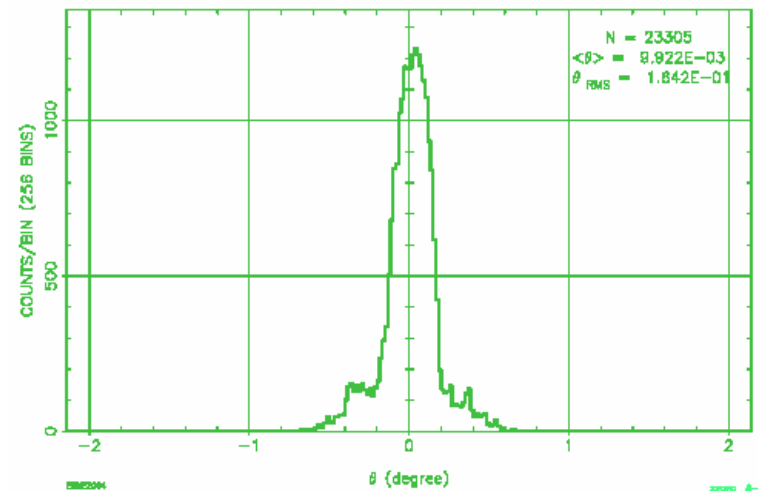

Fig. 1(c)

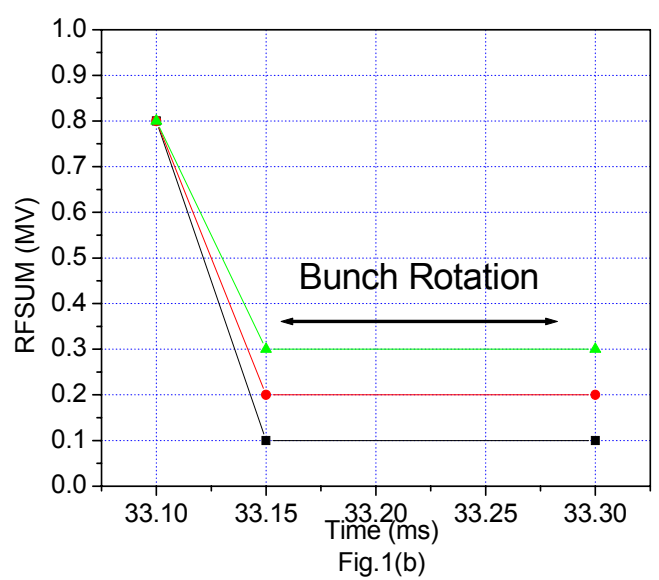

Itar 19600
$3.312 \mathrm{E}-02 \mathrm{SEC}$

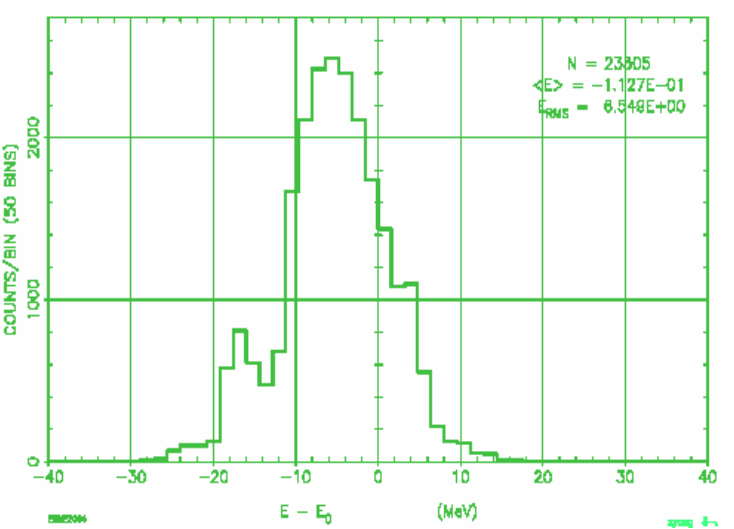

Fig. 1(d)

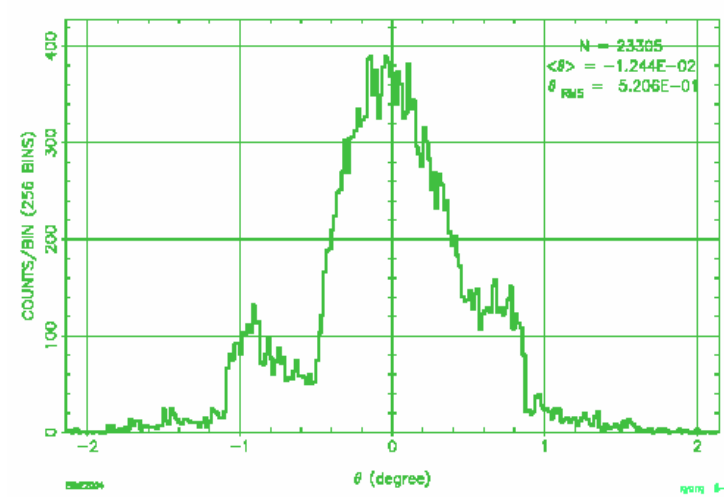

Fig. 1(e)

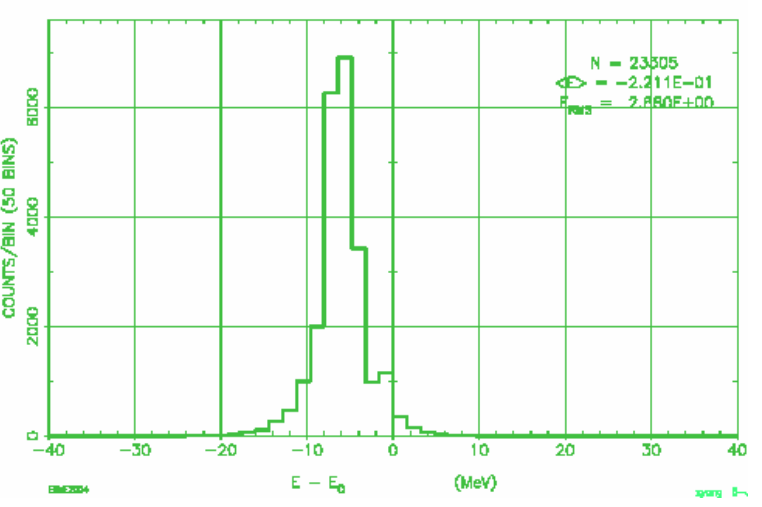

Fig. 1(f) 


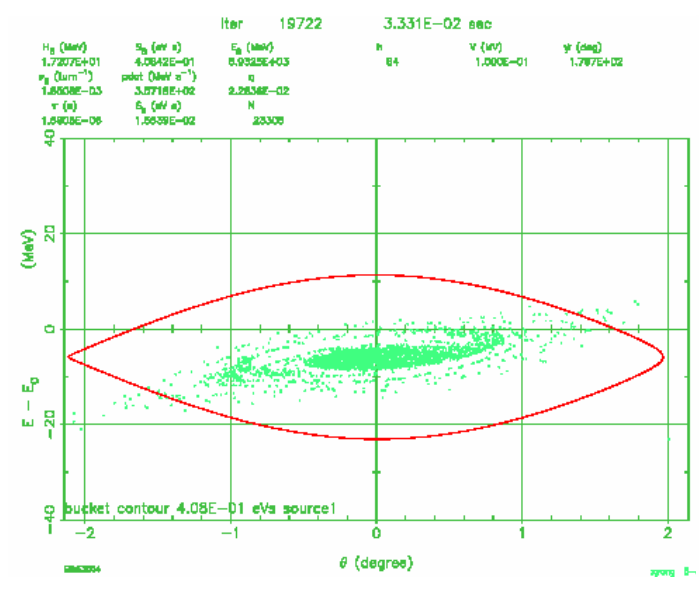

Fig. 1(g)

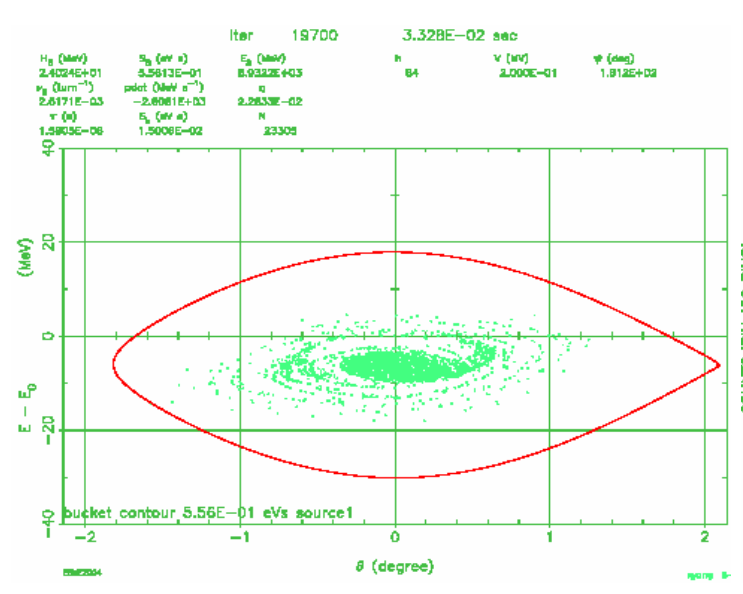

Fig. 1(i)

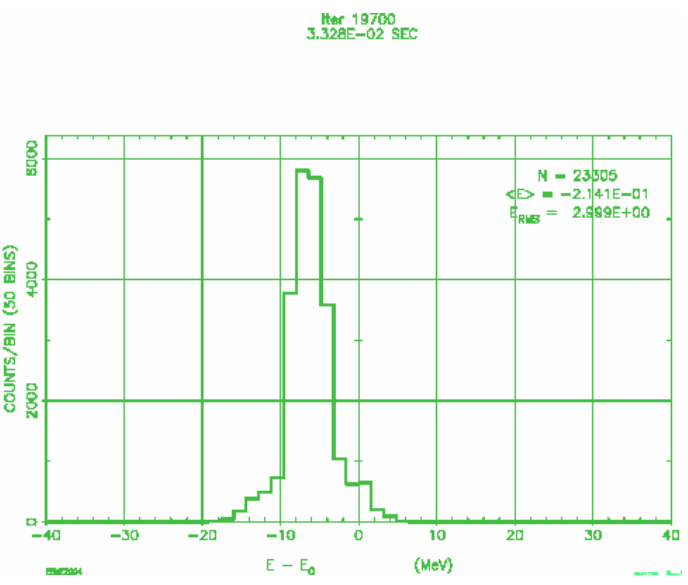

Fig. 1(h)

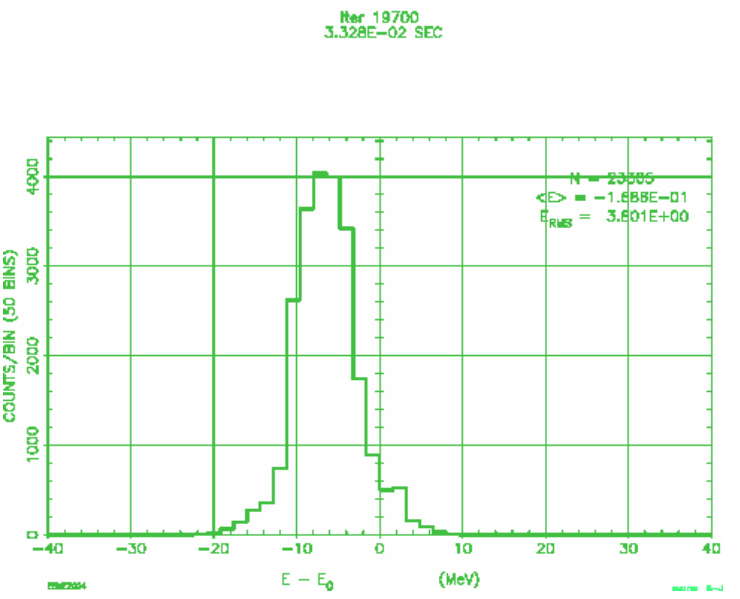

Fig.1(j)

Fig. 1(a) RFSUM vs. time in a Booster cycle. Three different BR voltages, $0.1 \mathrm{MV}, 0.2$ $\mathrm{MV}$, and $0.3 \mathrm{MV}$, are shown as the black, red, and green curves.

Fig. 1(b) the same with Fig. 1(a), except zooming into the BR time.

Fig. 1(c) the phase projection in the LPS before BR.

Fig. 1(d) the momentum projection in the LPS before BR.

Fig. 1(e) $V_{B R}=0.1 \mathrm{MeV}$, the phase projection at the optimal BR time.

Fig. 1(f) $V_{B R}=0.1 \mathrm{MeV}$, the momentum projection at the optimal BR time.

Fig. $1(\mathrm{~g}) V_{B R}=0.1 \mathrm{MeV}$, the particle distribution in the LPS at the optimal BR time.

Fig. 1(h) $V_{B R}=0.2 \mathrm{MeV}$, the momentum projection in the LPS at the optimal BR time.

Fig. 1(i) $V_{B R}=0.2 \mathrm{MeV}$, the particle distribution in the LPS at the optimal BR time.

Fig. 1(j) $V_{B R}=0.3 \mathrm{MeV}$, the momentum projection at the optimal BR time. 

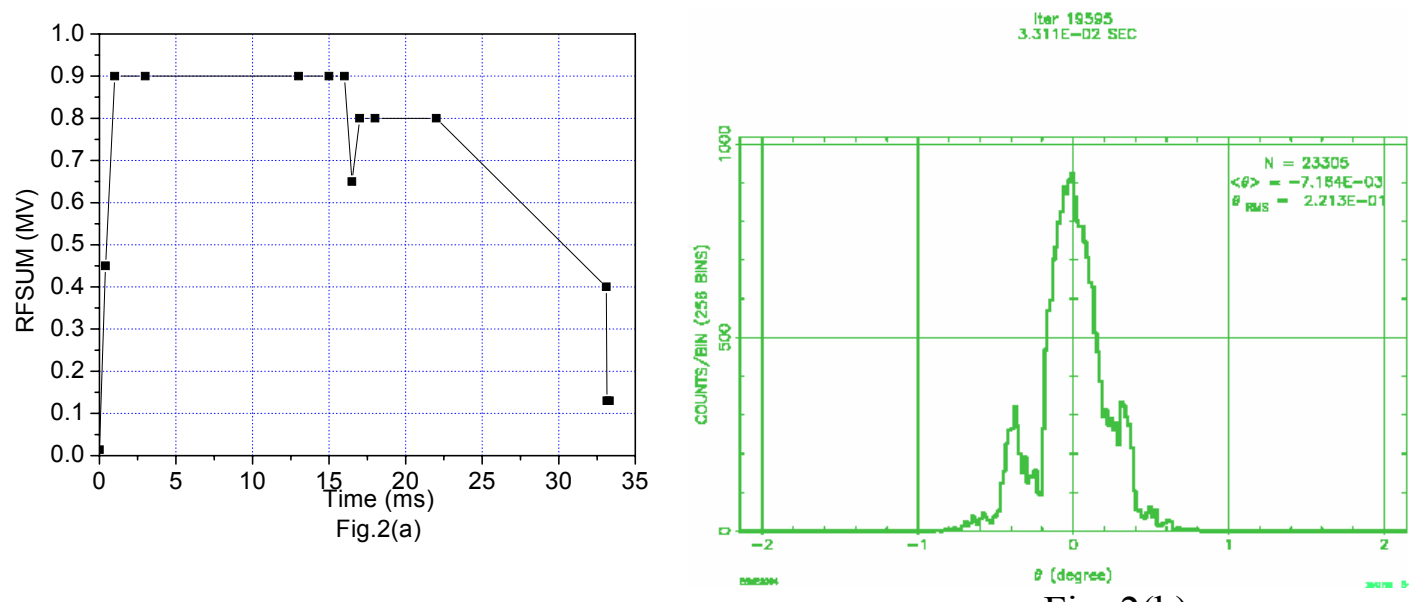

$\underset{\text { Fig. }}{\text { Fig.22 } 2(b)}$

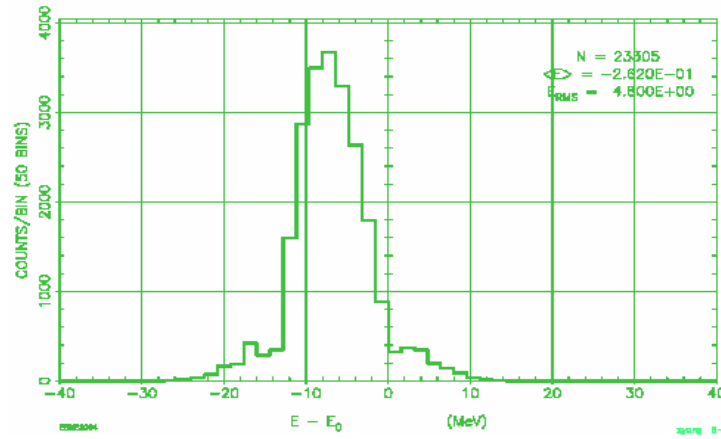

Fig. 2(c)

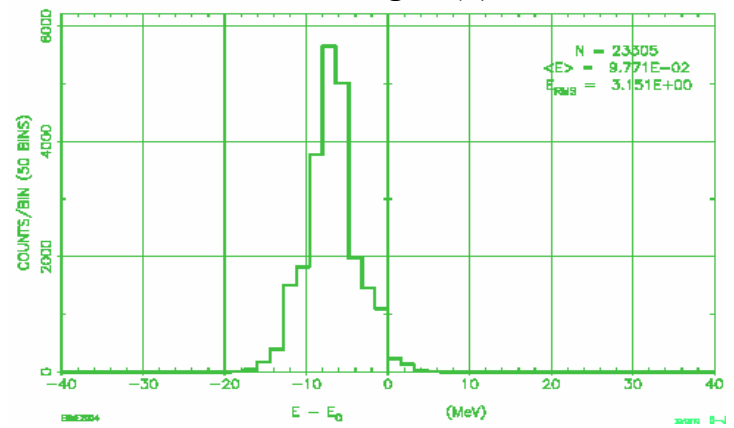

Fig. 2(e)

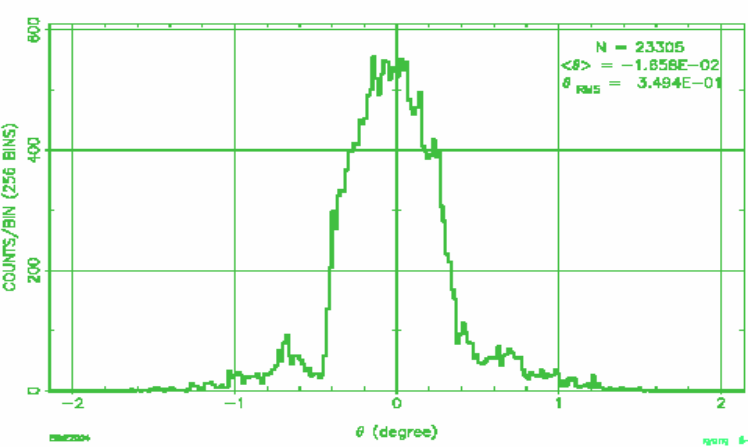

Fig. 2(d)

Fig. 2(a) RFSUM 2 vs. time in a Booster cycle.

Fig. 2(b) the phase projection of the particle distribution in the LPS right before BR.

Fig. 2(c) the momentum projection of the particle distribution in the LPS right before BR.

Fig. 2(d) $V_{B R}=0.13 \mathrm{MeV}$, the phase projection in the LPS at the optimal BR time.

Fig. 2(e) $V_{B R}=0.13 \mathrm{MeV}$, the momentum projection in the LPS at the optimal BR time. 

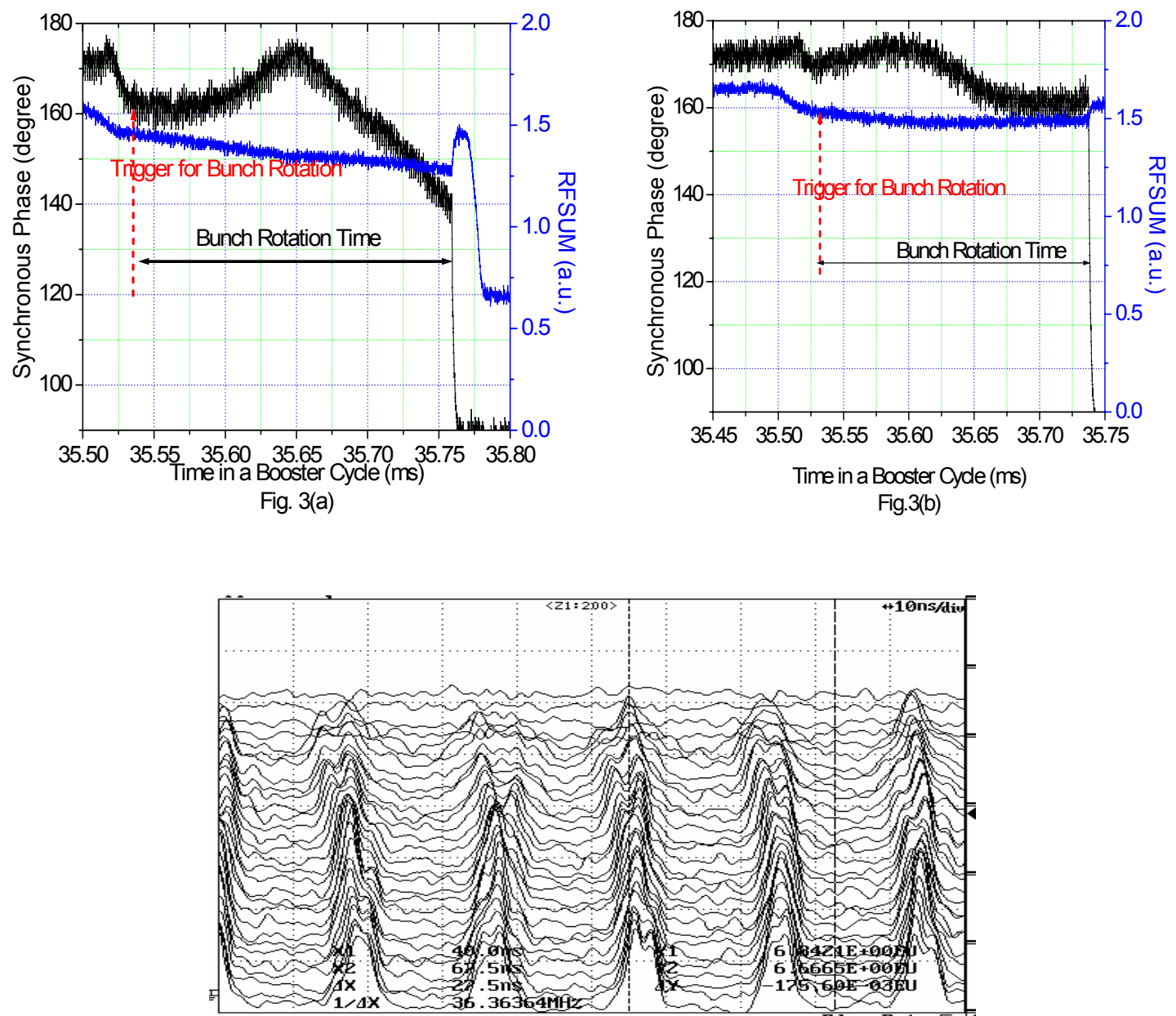

Fig. 3(c)

Fig. 3(a) in the situation that there was a LE growth after BR, the synchronous phase and RFSUM signals are shown as the black and blue curves respectively.

Fig. 3(b) in the situation that there was a negligible LE growth after BR, the synchronous phase and RFSUM signals are shown as the black and blue curves respectively.

Fig. 3(c) the MRP was taken at the same time with Fig. 3(b). The trigger time for the MRP is $35.4 \mathrm{~ms}$ in a Booster cycle, every 10 turns per trace. 


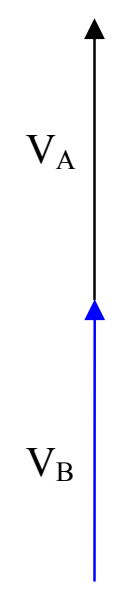

Fig. 4(a)

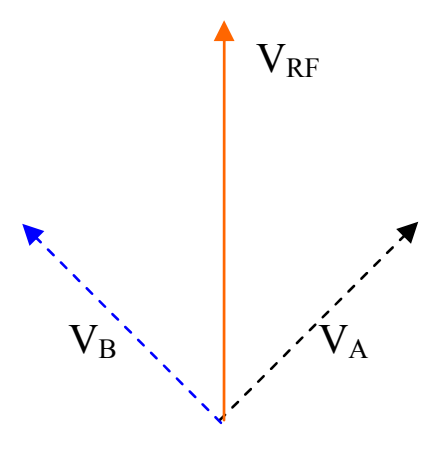

Fig. 4(b)

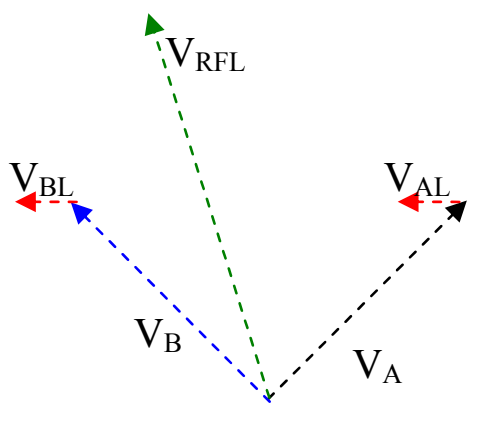

Fig. 4(c)

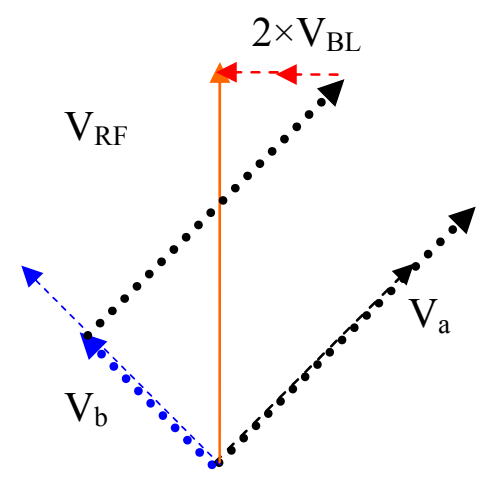

Fig. 4(d)

Fig. 4(a) RFSUM from A stations $\left(V_{A}\right)$ and RFSUM from $B$ stations $\left(V_{B}\right)$ before $B R$ are shown as the black and blue vectors.

Fig. 4(b) without considering the $B L, V_{A}$ and $V_{B}$ during $B R$ are shown as the dashed black and blue vectors, and RFSUM $\left(\mathrm{V}_{\mathrm{RF}}\right)$ is shown as the orange vector.

Fig. 4(c) taking the $\mathrm{BL}$ into consideration, the $\mathrm{BL}$ of $\mathrm{A}$ stations $\left(\mathrm{V}_{\mathrm{AL}}\right)$ and the $\mathrm{BL}$ of $\mathrm{B}$ stations $\left(\mathrm{V}_{\mathrm{BL}}\right)$ are represented by red dashed vectors, RFSUM during $\mathrm{BR}\left(\mathrm{V}_{\mathrm{RFL}}\right)$ is represented by the green vector.

Fig. 4(d) the amplitudes of $\mathrm{V}_{\mathrm{A}}$ and $\mathrm{V}_{\mathrm{B}}$ are adjusted via the $\mathrm{A}$ and $\mathrm{B}$ balancers to new values of $\mathrm{V}_{\mathrm{a}}$ and $\mathrm{V}_{\mathrm{b}}$, as shown by the dotted black and blue vectors, and the vector sum of $\mathrm{V}_{\mathrm{a}}, \mathrm{V}_{\mathrm{b}}, \mathrm{V}_{\mathrm{AL}}$, and $\mathrm{V}_{\mathrm{BL}}$ is shown as the orange vector. 